\title{
Comparison of anterior single- and standard two-portal techniques in arthroscopic Bankart repair
}

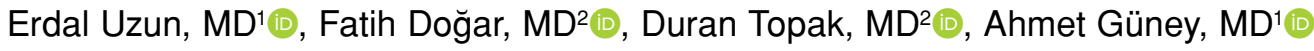 \\ 1Department of Orthopaedics and Traumatology, Erciyes University Medical Faculty, Kayseri, Turkey \\ ${ }^{2}$ Department of Orthopaedics and Traumatology, Kahramanmaraş Sütçü Imam University Medical Faculty, Kahramanmaraş, Turkey
}

Traumatic recurrent anterior shoulder instability is a common issue, particularly in young active patients. ${ }^{[1]}$ Repair of capsulolabral disruption has been considered the gold standard treatment for this condition. Although open Bankart repair is still used in the management of anterior shoulder instability, many arthroscopic surgical techniques have been developed over the past two decades, with increasing popularity providing a minimally invasive anatomical reconstruction with low operative complication rates. ${ }^{[2-5]}$

Arthroscopic Bankart repair (ABR) using one posterior and two anterior portals is the standard treatment for recurrent anterior shoulder instability, particularly in cases of isolated Bankart lesions or minimal loss of anterior glenoid rim. ${ }^{[6]}$ However, creating two anterior working portals is challenging in some populations, particularly in patients with small shoulders. ${ }^{[7]}$ Moreover, the standard anterior two-portal technique may lead to iatrogenic nerve

Received: October 27, 2020

Accepted: January 04, 2021

Published online: June 11, 2021

Correspondence: Fatih Doğar, MD. Kahramanmaraș Sütçü İmam Üniversitesi Tıp Fakültesi Ortopedi ve Travmatoloji Anabilim Dalı, 46050 Kahramanmaraş, Türkiye.

E-mail: drfatihdogar@hotmail.com

Doi: $10.52312 /$ jdrs.2021.79712

Citation: Uzun E, Doğar F, Topak D, Güney A. Comparison of anterior single- and standard two-portal techniques in arthroscopic Bankart repair. Jt Dis Relat Surg 2021;32(2):437-445.

(O2021 All right reserved by the Turkish Joint Diseases Foundation

This is an open access article under the terms of the Creative Commons Attribution-NonCommercial License, which permits use, distribution and reproduction in any medium, provided the original work is properly cited and is not used for commercial purposes (http://creativecommons.org/licenses/by-nc/4.0/).

\section{ABSTRACT}

Objectives: This study aims to compare the outcomes of patients undergoing a single anterior portal or a standard two-portal arthroscopic procedure for Bankart repair.

Patients and methods: Between January 2015 and March 2018, a total of 71 consecutive patients (53 males and 18 females; mean age: $33.3 \pm 10.3$ years; range, 17 to 56 years) who underwent arthroscopic Bankart repair with a minimum two-year follow-up period were included. The patients were divided into two groups according to the arthroscopic technique used: single anterior portal group (Group 1, $\mathrm{n}=32$ ) and standard two-portal group (Group 2, n=39). Demographic and surgical characteristics of the patients were recorded. Pre- and postoperative clinical and functional outcomes were evaluated using the external rotation degree, as well as Visual Analog Scale (VAS), American Shoulder and Elbow Surgeons (ASES), University of California at Los Angeles (UCLA) Shoulder Rating Scale, Constant-Murley Score (CMS), Oxford Shoulder Instability Score (OSIS), and Rowe scores. The clinical and functional outcomes and revision rates were compared between the groups.

Results: The mean follow-up was 32.0 \pm 7.4 months in Group 1

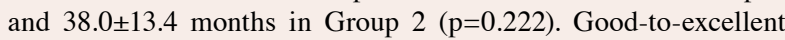
postoperative functional and clinical outcomes were achieved in both groups at the final follow-up, compared to baseline ( $p<0.001$ for all). No significant difference was observed in the postoperative outcomes including daily sports activity, VAS, ASES, UCLA Shoulder Rating Scale, CMS, OSIS, and Rowe scores, and external rotation restriction degrees between the groups $(p=0.270$, $\mathrm{p}=0.190, \mathrm{p}=0.313, \mathrm{p}=0.248, \mathrm{p}=0.125, \mathrm{p}=0.203, \mathrm{p}=0.318, \mathrm{p}=0.083$, respectively). The operative time in Group 1 was significantly lower than that in Group $2(60.3 \pm 8.3 v s .71 .4 \pm 7.2$, respectively; $\mathrm{p}=0.001)$. Four patients $(5.6 \%)$ experienced recurrent dislocation with no significant difference between the groups $(p=0.622)$. No significant complications occurred in the peri- or postoperative period. Fifty-eight $(81.7 \%)$ patients returned to their preoperative sports activity level. The mean time to return to sports was $7.2 \pm 1.7$ months.

Conclusion: Good-to-excellent clinical and functional outcomes can be obtained after arthroscopic Bankart repair, regardless of the use of a single or two anterior working portals. However, the single-portal technique is associated with reduced the operative time, compared to two-portal technique.

Keywords: Arthroscopy, Bankart lesion, joint instability, shoulder dislocation, surgical procedures. 
injuries and cannula breakage, as the cannulas have to be close to each other in small shoulders. ${ }^{[5,7]}$ Over the past five years, several studies have described the anterior single-portal technique for ABR. ${ }^{[2,8,9]}$ This technique is an effective ABR modality with similar outcomes as that of the anterior two-portal technique; however, few studies have compared the two techniques. ${ }^{[5,10,11]}$ In this study, we hypothesized that the results of $A B R$ might vary based on the number of working portals used. We, therefore, aimed to compare the clinical and functional outcomes and revision rates of patients who underwent $A B R$ using two different techniques: a single anterior portal and two standard portals.

\section{PATIENTS AND METHODS}

This multi-center, retrospective study was conducted at Kahramanmaraş Sütçü Imam University Medical Faculty and Erciyes University Medical Faculty, Department of Orthopaedics and Traumatology between January 2015 and March 2018. A total of 119 patients who underwent ABR for traumatic anterior shoulder instability were screened. A surgical team with at least five years of experience performing arthroscopic shoulder surgery performed the surgeries for all patients. The data of 71 consecutive patients ( 53 males and 18 females; mean age: $33.3 \pm 10.3$ years; range, 17 to 56 years) who underwent ABR with a minimum follow-up period of two years were retrospectively analyzed. The patients were divided into two groups based on the arthroscopic technique performed: Group
1, single anterior portal $(\mathrm{n}=32)$ and Group 2, two standard portals $(n=39)$. Inclusion criteria were as follows: patients with primary arthroscopic shoulder stabilization, anterior traumatic instability with or without a concomitant superior labrum anterior and posterior (SLAP) ${ }^{[8]}$ lesion, and Bankart or Bankart-like lesions (Perthes, anterior, labroligamentous periosteal sleeve avulsion [ALPSA]) lesion with/without a minimal Hill-Sachs lesion requiring $\mathrm{ABR}$; those willing to participate in the study; those compliant with the postoperative rehabilitation protocol; and those having a minimum follow-up period of two years. Exclusion criteria were as follows: having multidirectional instability without an explicit traumatic episode $(n=4)$; the presence of preoperative arthropathy according to the Samilson-Prieto classification ${ }^{[9]}$ which categorizes osteoarthritis (OA) in four categories comprising (i) no OA, (ii) osteophytes measuring $<3 \mathrm{~mm}$ in greatest distance diameter, (iii) osteophytes measuring between 3 and $7 \mathrm{~mm}$ in greatest distance diameter and slight glenohumeral joint irregularity, and (iv) osteophytes measuring $>7 \mathrm{~mm}$ in greatest distance diameter, narrowing of the glenohumeral joint and sclerosis) $(n=1)$; preoperative rotator cuff injury $(\mathrm{n}=2)$; a history of ipsilateral upper extremity surgery $(n=3)$; posterior instability repair $(n=2)$; rheumatic disease $(\mathrm{n}=2)$; neurological problems including cervical myelopathy or any neuropathic disorder affecting the ipsilateral or contralateral extremity $(n=3)$; previous surgery for the affected shoulder $(n=6)$;

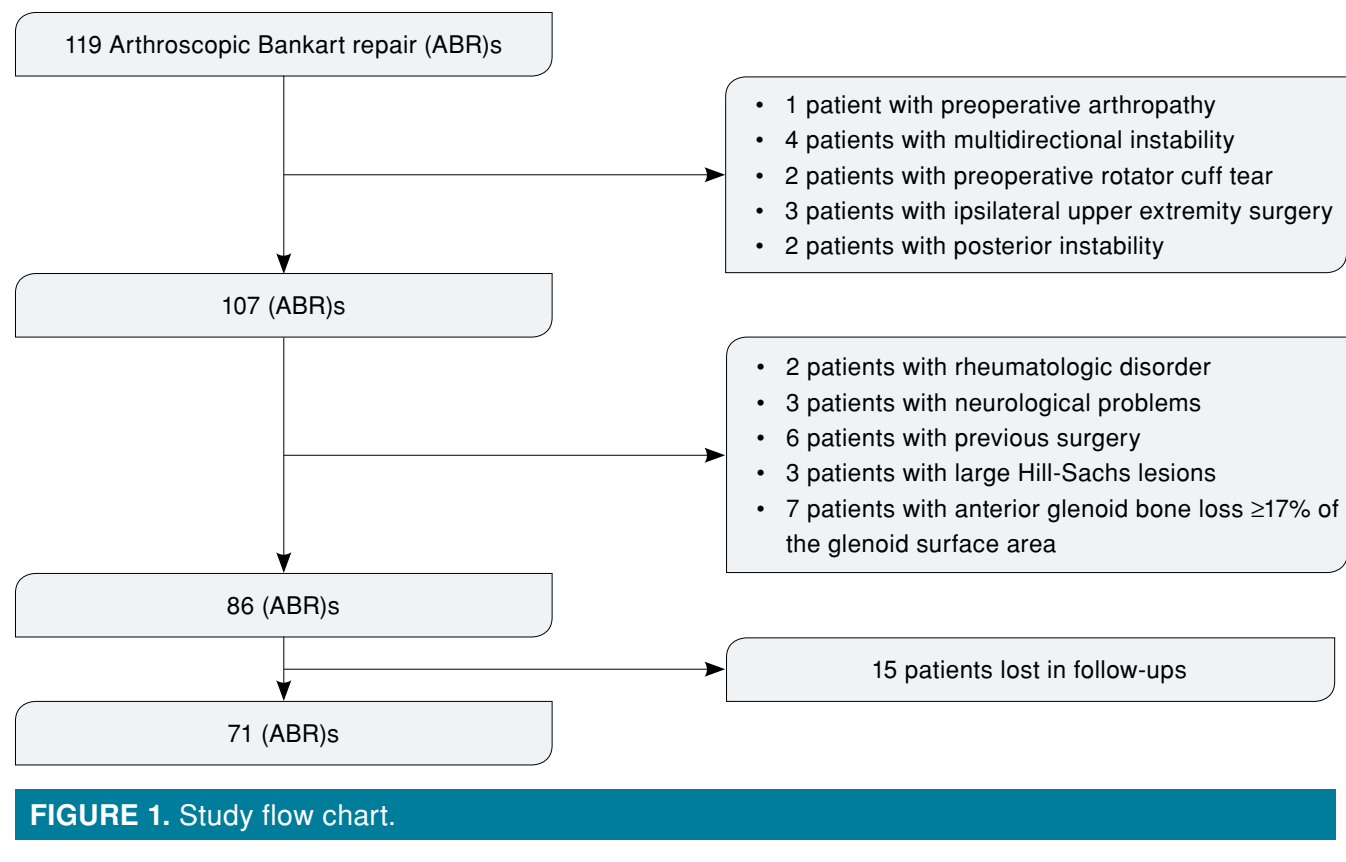


large Hill-Sachs lesions ( $\mathrm{n}=3$ ); anterior glenoid bone loss $>17 \%$ of the glenoid surface area ${ }^{[10]}(n=7)$; and loss to follow-up ( $n=15)$. The study flow chart is shown in Figure 1. A written informed consent was obtained from each participant. The study protocol was approved by the Kahramanmaraş Sütçü Imam University Medical Faculty Ethics Committee (Date: 29.04.2020; No: 2020/15). The study was conducted in accordance with the principles of the Declaration of Helsinki.

\section{Surgical method}

All surgical procedures were performed arthroscopically in a beach chair position under general anesthesia. Diagnostic arthroscopy was conducted using a standard posterior portal. In Group 1, a second single anterior working portal was established through the rotator interval nearly $1 \mathrm{~cm}$ superior and $1 \mathrm{~cm}$ lateral to the coracoid notch (the best position and angle was determined to allow access to the anteroinferior capsule and glenoid labrum by means of an epidural needle with outside-in technique. The lateral aspect along the superior border of the subscapularis tendon in the appropriate trajectory was the entry point of the needle) (Figure 2a). In Group 2, standard anterosuperior and anteroinferior portals were created. The cannula was inserted in the anterior portal in both groups. After the anteroinferior capsulolabral complex was completely detached from the glenoid (Figure 2b, c), capsulolabral repair was performed through the anterior portal. For single-portal repair technique first a suture transferring system (ACCU-PASS Suture Shuttle, Smith and Nephew, UK) was used to pass the anterior capsulolabral complex taking a healthy bite of the labrum and capsules distally as possible, and further an ample amount of the non-degradable suture of suture shuttle was left in the joint (Figure 3a). The suture shuttle was, then, withdrawn from the anterior portal and a grasper was used to retrieve the transferring suture passed through the labrum (Figure 3b, c). A 1.7-mm, single-loaded suture anchor (SUTUREFIX, Smith and Nephew, London, UK) was placed through the anterior glenohumeral portal (Figure 4a). The first anchor was inserted inferiorly as close as the 5.30 position or 6.30 position in the right and left shoulders, respectively (Figure $4 b$ ). Subsequently, one end of the anchor threads was transferred and passed through the capsulolabral complex using the transferring suture (Figure 4c). The sutures were, then, simply tied to fix the capsulolabral complex on the anterior glenoid rim (Figure $4 \mathrm{~d}$ ). Depending on the extent of labrum detachment from the glenoid rim and the presence of concomitant SLAP lesion, two or more anchors were used to complete the repair of the capsulolabral complex. Threads of other additional anchors were transferred and subsequently fixated in a similar manner at an appropriate position. Bankart repair was performed similarly to ALPSA $(n=5)$ or Perthes $(n=2)$ lesions. Six patients had concomitant SLAP lesions: four patients with Type I SLAP lesion were treated with debridement and two patients with Type V SLAP lesion underwent SLAP repair in addition to ABR.

\section{Postoperative rehabilitation}

All patients remained in a shoulder immobilizer in slight abduction and neutral positions for six weeks postoperatively. For the first six weeks, pendulum exercises to flex the elbow and wrist
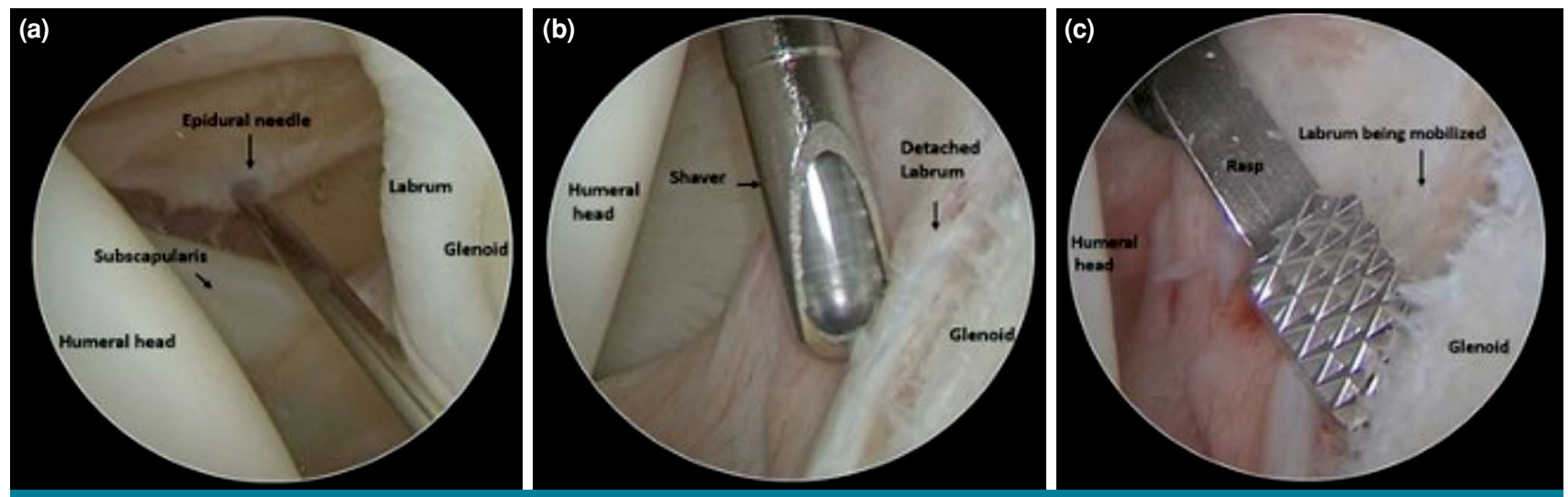

FIGURE 2. Arthroscopic view from the posterior portal of the left shoulder. (a) Establishment of single anterior working portal through the rotator interval by means of an epidural needle along the superior border of the subscapularis tendon with outside-in technique. (b) The view of the detached labrum and Bankart lesion. (c) The anterior glenoid neck being prepared with a rasp and anterior labrum mobilized. 

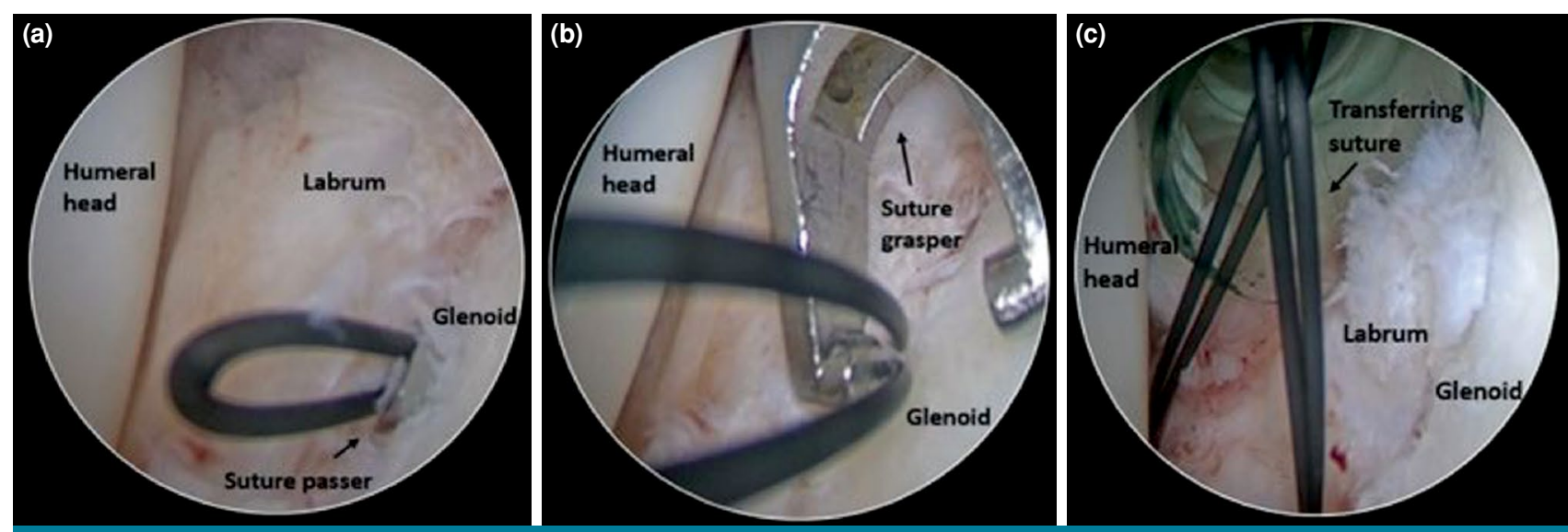

FIGURE 3. Single anterior portal technique, arthroscopic view from the posterior portal of the left shoulder. (a) A suture transferring system passing through the detached labrum and further lefting an ample amount of the suture in the joint. (b, c) After withdrawn of the suture shuttle from the anterior portal, a grasper is used to retrieve the transferring suture.

were allowed; in the subsequent six weeks, active assisted/passive range of motion (ROM) exercises were initiated. After three months, strengthening and resistance exercises were initiated. Return to sports activities were permitted after four months, whereas contact sports were allowed six to nine months after surgery.

\section{Assessments}

The demographic characteristics of the patients were recorded from the prospectively collected data. A standard physical examination was conducted to record ROM pre- and postoperatively. For radiological evaluation, standard anteroposterior and axillary views were used. Preoperative axial magnetic resonance imaging (MRI) scans were used to identify the Hill-Sachs lesions and glenoid defects. The patients with glenoid defects on MRI underwent a three-dimensional computed tomography scan, and the defect size was measured by a physician not participating in the surgery according to the method described by Sugaya et al. ${ }^{[12]}$ Hill-Sachs lesions were intraoperatively evaluated and subjectively described as small, medium, or large. Deep lesions involving more than $15 \%$ of the humeral articular surface were defined as large lesions. For small- and medium-sized lesions, the remplissage procedure was performed following labral repair, if signs of instability persisted in arthroscopic control. Routine follow-up was performed at 1.5, three, six, and 12 months and annually, thereafter. Operative reports were assessed and pre- and postoperative (at the final follow-up) clinical and functional evaluations were performed. Pre- and postoperative pain and clinical and functional outcomes were measured using the following parameters: the external rotation degree was calculated by comparing the ROM of the patient's affected side with that of the patient's healthy side; the Visual Analog Scale (VAS), with scores ranging from 0 to 10 (0: no pain; 10: worst pain ever); the American Shoulder and Elbow Surgeons (ASES) Standardized Shoulder Assessment Form; ; ${ }^{[13]}$ the University of California at Los Angeles (UCLA)
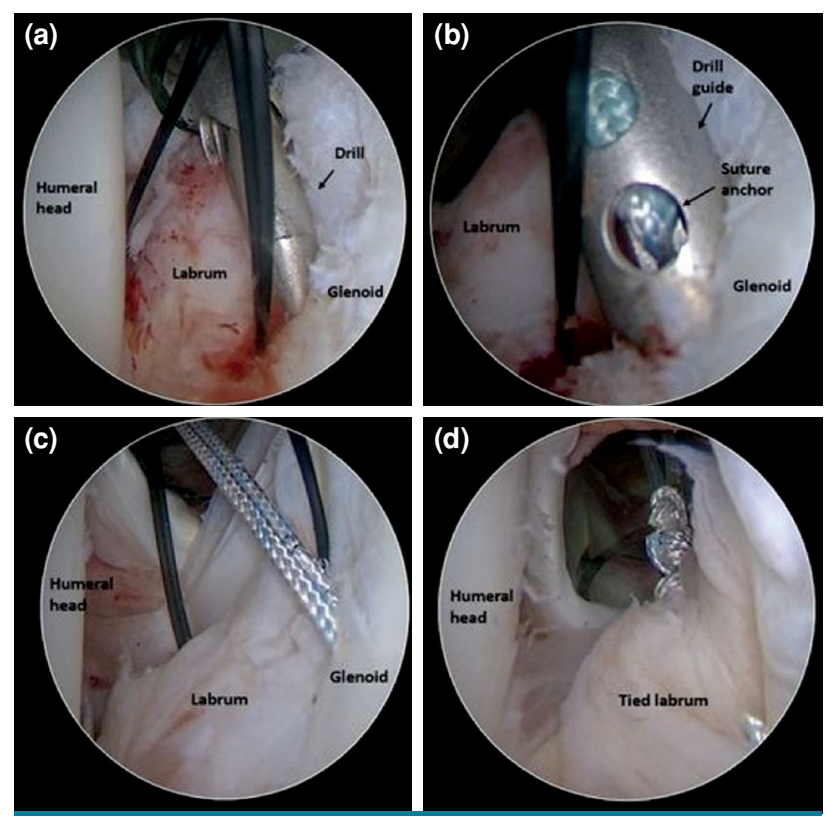

FIGURE 4. Arthroscopic view from the posterior portal of the left shoulder. (a) Preparation a pilot hole for the suture anchor with drill through the anterior portal. (b) Placing the anchor under the guidance of the drill guide. (c) The one end of the anchor threads transferred and passed through the capsulolabral complex using the transferring suture. (d) The suture threads simply tied to fix the capsulolabral complex on the anterior glenoid rim. 


\begin{tabular}{|c|c|c|c|c|c|c|c|c|c|c|c|c|c|}
\hline & & & Patient cha & acteristic & $\begin{array}{l}\text { TA } \\
\text { in } s\end{array}$ & $\begin{array}{l}\text { LE I } \\
\text { gle- ar }\end{array}$ & d two-porta & groups & & & & & \\
\hline & & & otal $(n=71)$ & & & gle ant & erior portal & $n=32)$ & & ble an & erior portals & $(n=39)$ & \\
\hline & $\mathrm{n}$ & $\%$ & Mean \pm SD & Range & $\mathrm{n}$ & $\%$ & Mean $\pm S D$ & Range & $\mathrm{n}$ & $\%$ & Mean $\pm S D$ & Range & $p$ \\
\hline Age at surgery (year) & & & $33.3 \pm 10.3$ & $17-56$ & & & $36.0 \pm 11.2$ & $17-56$ & & & $31.0 \pm 9.0$ & $18-55$ & 0.062 \\
\hline Age at first dislocation (year) & & & $31.4 \pm 10.1$ & $16-54$ & & & $33.7 \pm 11.2$ & $16-54$ & & & $29.5 \pm 8.9$ & $18-53$ & 0.124 \\
\hline Sex & & & & & & & & & & & & & 0.113 \\
\hline Female & 18 & 25.9 & & & 11 & 34.4 & & & 7 & 17.9 & & & \\
\hline Male & 53 & 74.6 & & & 21 & 65.6 & & & 32 & 82.1 & & & \\
\hline Side & & & & & & & & & & & & & 0.349 \\
\hline Right & 42 & 59.2 & & & 17 & 53.1 & & & 24 & 64.1 & & & \\
\hline Left & 29 & 40.8 & & & 15 & 46.9 & & & 14 & 35.9 & & & \\
\hline Side dominancy & & & & & & & & & & & & & 0.486 \\
\hline No & 23 & 32.4 & & & 9 & 28.1 & & & 14 & 35.9 & & & \\
\hline Yes & 48 & 67.6 & & & 23 & 71.9 & & & 25 & 64.1 & & & \\
\hline $\mathrm{BMI}\left(\mathrm{kg} / \mathrm{m}^{2}\right)$ & & & $25.3 \pm 2.9$ & & & & $25.9 \pm 2.8$ & & & & $24.9 \pm 2.9$ & & 0.153 \\
\hline Contact sports & & & & & & & & & & & & & 0.086 \\
\hline- & 32 & 45.1 & & & 18 & 56.2 & & & 14 & 35.9 & & & \\
\hline+ & 39 & 54.9 & & & 14 & 43.8 & & & 25 & 64.1 & & & \\
\hline Smoking habit & & & & & & & & & & & & & 0.817 \\
\hline- & 41 & 57.7 & & & 18 & 56.2 & & & 23 & 59.0 & & & \\
\hline+ & 30 & 42.3 & & & 14 & 43.8 & & & 16 & 41.0 & & & \\
\hline Comorbidity* & & & & & & & & & & & & & 0.231 \\
\hline- & 64 & 90.1 & & & 27 & 84.4 & & & 37 & 94.9 & & & \\
\hline+ & 7 & 9.9 & & & 5 & 15.6 & & & 2 & 5.1 & & & \\
\hline
\end{tabular}

\begin{tabular}{|c|c|c|c|c|c|c|c|c|c|c|c|c|c|}
\hline & cal c & aracte & istics and if & jury patt & $\begin{array}{l}\text { TAB } \\
\text { ns of }\end{array}$ & $\begin{array}{l}\text { E II } \\
\text { all pat }\end{array}$ & nts and sin & le- and & $0-p$ & tal gro & ups & & \\
\hline & & & tal $(n=71)$ & & & gle an & erior portal & n=32) & & ble an & erior portals & $(n=39)$ & \\
\hline & $\mathrm{n}$ & $\%$ & Mean $\pm S D$ & Range & $n$ & $\%$ & Mean $\pm S D$ & Range & $\mathrm{n}$ & $\%$ & Mean $\pm S D$ & Range & $p$ \\
\hline Type of the injury & & & & & & & & & & & & & 0.365 \\
\hline Training & 31 & 43.7 & & & 12 & 37.5 & & & 19 & 48.7 & & & \\
\hline Sports & 35 & 49.3 & & & 16 & 50.0 & & & 19 & 48.7 & & & \\
\hline Fall & 4 & 5.6 & & & 3 & 9.4 & & & 1 & 2.6 & & & \\
\hline Accident & 1 & 1.4 & & & 1 & 3.1 & & & 0 & 0.0 & & & \\
\hline $\begin{array}{l}\text { Number of instability } \\
\text { episodes }\end{array}$ & & & & & & & & & & & & & 0.463 \\
\hline$\geq 5$ & 30 & 42.3 & & & 12 & 37.5 & & & 18 & 46.2 & & & \\
\hline$<5$ & 41 & 57.7 & & & 20 & 62.5 & & & 21 & 53.8 & & & \\
\hline $\begin{array}{l}\text { Time from first dislocation } \\
\text { to operation (month) }\end{array}$ & & & $17.8 \pm 12.8$ & $1-80$ & & & $20.8 \pm 16.1$ & $6-80$ & & & $15.3 \pm 8.9$ & $4-60$ & 0.259 \\
\hline No. of suture anchors & & & $2.6 \pm 0.5$ & $2-4$ & & & $2.7 \pm 0.5$ & $2-4$ & & & $2.6 \pm 0.5$ & $2-4$ & 0.531 \\
\hline Glenoid bone loss & & & & & & & & & & & & & 0.446 \\
\hline$(-)$ & 64 & 90.1 & & & 30 & 93.7 & & & 34 & 87.2 & & & \\
\hline$(+)$ & 7 & 9.9 & & & 2 & 6.3 & & & 5 & 12.8 & & & \\
\hline Hill-Sachs lesion & & & & & & & & & & & & & 0.814 \\
\hline$(-)$ & 52 & 73.2 & & & 23 & 71.9 & & & 29 & 74.4 & & & \\
\hline Small/medium & 19 & 26.8 & & & 9 & 28.1 & & & 10 & 25.6 & & & \\
\hline SLAP lesion & & & & & & & & & & & & & 0.683 \\
\hline No & 65 & 91.5 & & & 30 & 93.8 & & & 35 & 89.7 & & & \\
\hline Yes & 6 & 8.5 & & & 2 & 6.3 & & & 4 & 10.3 & & & \\
\hline Perthes lesion & & & & & & & & & & & & & 0.887 \\
\hline No & 69 & 97.2 & & & 31 & 96.9 & & & 38 & 97.4 & & & \\
\hline Yes & 2 & 2.8 & & & 1 & 3.1 & & & 1 & 2.6 & & & \\
\hline ALPSA lesion & & & & & & & & & & & & & 0.813 \\
\hline No & 66 & 93.0 & & & 30 & 93.8 & & & 36 & 92.3 & & & \\
\hline Yes & 5 & 7.0 & & & 2 & 6.3 & & & 3 & 7.7 & & & \\
\hline Operative time (minutes) & & & $65.3 \pm 9.5$ & $45-85$ & & & $60.3 \pm 8.3$ & $45-75$ & & & $71.4 \pm 7.2$ & $55-85$ & 0.000 \\
\hline
\end{tabular}


Shoulder Rating Scale, ${ }^{[14]}$ the Constant-Murley Score (CMS), ${ }^{[15]}$ the Oxford Shoulder Instability Score (OSIS); ${ }^{[16]}$ and the Rowe score. ${ }^{[17]}$ We did not perform routine postoperative MRI. To assess the postoperative recurrent instability, the patients were asked whether they experienced a redislocation after surgery and they needed help to have the shoulder reduced; those who answered "Yes" to both questions were considered to have a dislocation. The demographic data and functional and clinical outcome parameters were compared between the two groups.

\section{Statistical analysis}

Statistical analysis was performed using the IBM SPSS for Windows version 22.0 software (IBM Corp., Armonk, NY, USA). Descriptive data were expressed in mean \pm standard deviation (SD), median (min-max) or number and frequency, where applicable. The independent samples t-test and Mann-Whitney $U$ test were used for analyzing independent quantitative data, Wilcoxon's test for dependent quantitative data, and the chi-square test for independent qualitative data. The Fisher's

\begin{tabular}{|c|c|c|c|c|c|c|c|c|c|c|}
\hline \multirow[b]{3}{*}{ Evaluation method } & \multicolumn{9}{|c|}{$\begin{array}{l}\text { TABLE III } \\
\text { ee scores in }\end{array}$} & \multirow[b]{3}{*}{$p$} \\
\hline & \multicolumn{3}{|c|}{ Total $(n=71)$} & \multicolumn{3}{|c|}{ Single anterior portal $(n=32)$} & \multicolumn{3}{|c|}{ Double anterior portals $(n=39)$} & \\
\hline & $\mathrm{n}$ & $\%$ & Mean $\pm S D$ & $\mathrm{n}$ & $\%$ & Mean $\pm S D$ & $\mathrm{n}$ & $\%$ & Mean $\pm S D$ & \\
\hline Follow-up period (month) & & & $35.3 \pm 11.5$ & & & $32.0 \pm 7.4$ & & & $38.0 \pm 13.4$ & 0.222 \\
\hline Length of hospital stay (day) & & & $1.28 \pm 0.48$ & & & $1.2 \pm 0.4$ & & & $1.41 \pm 0.56$ & 0.059 \\
\hline $\begin{array}{l}\text { Daily sports activity }(\mathrm{h}) \\
\text { Preoperative } \\
\text { Postoperative } \\
p\end{array}$ & & & $\begin{array}{l}0.8 \pm 0.4 \\
1.5 \pm 0.7 \\
<0.001\end{array}$ & & & $\begin{array}{l}0.8 \pm 0.4 \\
1.4 \pm 0.7 \\
<0.001\end{array}$ & & & $\begin{array}{l}0.9 \pm 0.4 \\
1.5 \pm 0.6 \\
<0.001\end{array}$ & $\begin{array}{l}0.400 \\
0.270\end{array}$ \\
\hline $\begin{array}{l}\text { VAS score } \\
\text { Preoperative } \\
\text { Postoperative } \\
p\end{array}$ & & & $\begin{array}{l}4.6 \pm 1.3 \\
1.0 \pm 1.0 \\
<0.001\end{array}$ & & & $\begin{array}{l}4.5 \pm 1.6 \\
0.9 \pm 1.0 \\
<0.001\end{array}$ & & & $\begin{array}{l}4.8 \pm 1.0 \\
1.2 \pm 1.0 \\
<0.001\end{array}$ & $\begin{array}{l}0.302 \\
0.190\end{array}$ \\
\hline $\begin{array}{l}\text { ASES score } \\
\text { Preoperative } \\
\text { Postoperative } \\
p\end{array}$ & & & $\begin{array}{l}61.6 \pm 6.8 \\
87.4 \pm 10.8 \\
<0.001\end{array}$ & & & $\begin{array}{l}60.3 \pm 6.3 \\
88.4 \pm 11.3 \\
<0.001\end{array}$ & & & $\begin{array}{l}62.7 \pm 7.1 \\
86.6 \pm 10.5 \\
<0.001\end{array}$ & $\begin{array}{l}0.186 \\
0.313\end{array}$ \\
\hline $\begin{array}{l}\text { UCLA score } \\
\text { Preoperative } \\
\text { Postoperative } \\
p\end{array}$ & & & $\begin{array}{c}18.7 \pm 3.5 \\
31.5 \pm 4.4 \\
<0.001\end{array}$ & & & $\begin{array}{c}19.3 \pm 2.9 \\
30.8 \pm 4.7 \\
<0.001\end{array}$ & & & $\begin{array}{c}18.3 \pm 3.8 \\
32.2 \pm 4.1 \\
<0.001\end{array}$ & $\begin{array}{l}0.446 \\
0.248\end{array}$ \\
\hline $\begin{array}{l}\text { CM score } \\
\text { Preoperative } \\
\text { Postoperative } \\
p\end{array}$ & & & $\begin{array}{c}56.6 \pm 7.1 \\
89.7 \pm 10.5 \\
<0.001\end{array}$ & & & $\begin{array}{c}55.9 \pm 6.5 \\
88.3 \pm 9.8 \\
<0.001\end{array}$ & & & $\begin{array}{c}57.2 \pm 7.5 \\
90.9 \pm 11.1 \\
<0.001\end{array}$ & $\begin{array}{l}0.299 \\
0.125\end{array}$ \\
\hline $\begin{array}{l}\text { Oxford instability score } \\
\text { Preoperative } \\
\text { Postoperative } \\
p\end{array}$ & & & $\begin{array}{c}23.2 \pm 4.9 \\
43.0 \pm 6.1 \\
<0.001\end{array}$ & & & $\begin{array}{c}23.0 \pm 6.1 \\
43.6 \pm 6.2 \\
<0.001\end{array}$ & & & $\begin{array}{c}23.4 \pm 3.8 \\
42.5 \pm 6.1 \\
<0.001\end{array}$ & $\begin{array}{l}0.310 \\
0.203\end{array}$ \\
\hline $\begin{array}{l}\text { Rowe score } \\
\text { Preoperative } \\
\text { Postoperative } \\
p\end{array}$ & & & $\begin{array}{c}51.1 \pm 9.4 \\
89.6 \pm 11.5 \\
<0.001\end{array}$ & & & $\begin{array}{c}50.6 \pm 8.2 \\
88.7 \pm 10.7 \\
<0.001\end{array}$ & & & $\begin{array}{l}51.5 \pm 10.5 \\
90.3 \pm 12.3 \\
<0.001\end{array}$ & $\begin{array}{l}0.805 \\
0.318\end{array}$ \\
\hline $\begin{array}{l}\text { External rotation restriction } \\
\text { Preoperative } \\
\text { Postoperative } \\
p\end{array}$ & & & $\begin{array}{l}16.6 \pm 6.2 \\
8.5 \pm 6.4 \\
<0.001\end{array}$ & & & $\begin{array}{l}18.2 \pm 5.3 \\
7.0 \pm 5.9 \\
<0.001\end{array}$ & & & $\begin{array}{l}15.3 \pm 6.6 \\
9.7 \pm 6.5 \\
<0.001\end{array}$ & $\begin{array}{l}0.065 \\
0.083\end{array}$ \\
\hline $\begin{array}{l}\text { Recurrent instability } \\
\text { No } \\
\text { Yes }\end{array}$ & $\begin{array}{c}67 \\
4\end{array}$ & $\begin{array}{c}94.4 \\
5.6\end{array}$ & & $\begin{array}{c}31 \\
1\end{array}$ & $\begin{array}{c}96.6 \\
3.1\end{array}$ & & $\begin{array}{c}36 \\
3\end{array}$ & $\begin{array}{c}92.3 \\
7.7\end{array}$ & & 0.622 \\
\hline
\end{tabular}


exact test was used, when the chi-square test requirements were not met. A $p$ value of $<0.05$ was considered statistically significant.

\section{RESULTS}

The mean follow-up period was $35.3 \pm 11.5$ months (range, 24-60). Of the 71 patients, 32 (45.1\%) underwent ABR with the single anterior portal technique (Group 1) and 39 (54.9\%) underwent ABR with the two-portal technique (Group 2). No significant differences were observed between the groups in terms of patients' characteristics and demographic data (Table I). Additionally, injury patterns and surgical characteristics, except for operative time, were similar between the groups (Table II). Operative time was significantly lower in Group 1 than in Group 2 (60.3 \pm 8.3 vs. $71.4 \pm 7.2 ; p=0.000)$. In both groups, patients demonstrated a significant pre- to postoperative improvement in clinical and functional outcome scores $(\mathrm{p}<0.001)$; however, this improvement was not significantly different between the groups. Furthermore, the length of hospital stay and recurrent instability rates were not statistically significantly different between the groups $(\mathrm{p}<0.05)$. The clinical and functional outcome scores are summarized in Table III. The overall recurrent instability rate at the final follow-up was $5.6 \%(4 / 71)$. One patient $(3.1 \%)$ in Group 1 and three patients (7.7\%) in Group 2 with recurrence underwent revision $A B R(p=0.622)$. After the revision surgery, no redislocations occurred during the remaining follow-up period. Twenty-six patients (81.3\%) in Group 1 and 32 patients (82.1\%) in Group 2 returned to their preoperative sports activity level $(\mathrm{p}=0.931)$. The mean time to return to preoperative sports activity was $7.5 \pm 1.7$ and 7.0 \pm 1.7 months in Groups 1 and Group 2, respectively $(p=0.079)$. No patients developed superficial or deep infections, and no significant complications occurred in the peri- or postoperative period.

\section{DISCUSSION}

The main finding of this study is that the anterior single-portal technique is as effective, reliable, and reproducible as the standard two-portal technique, with similar good-to-excellent clinical and functional outcomes. Furthermore, compared to the standard two-portal technique, the anterior single-portal technique was associated with short operative time and low rate of neurovascular injuries.

Over the past two decades, several arthroscopic techniques have been developed, particularly for shoulder surgery. ${ }^{[2-5,18,19]}$ The advantages of ABR are as follows: less postoperative pain, less loss of motion, shorter length of hospital stay, lower morbidity rates, shorter operative time, and better cosmetic appearance. ${ }^{[20]}$ Previously, the standard anterior two-portal technique was used in ABR surgery; however, the single anterior portal technique, which is less invasive, has been introduced and increasingly used over the past five years. ${ }^{[2,5,11,18-20-22]}$ This present study evaluated the effectiveness of single anterior portal technique as an up-to-date treatment method in ABR. A detailed evaluation was performed using clinical and functional outcome measures and recurrence rates. Compared to the standard two anterior portal, single-portal technique showed similar outcomes concurrent with the literature. ${ }^{[5,11,20]}$ Moreover, the results of the current study showed a similar recurrence rate with a single anterior portal, compared to the standard two-portal technique after at least two years.

Few studies have compared the results of the single anterior portal and standard two-portal techniques. ${ }^{[5,11,20]}$ Cicek et al. ${ }^{[20]}$ compared ABR with the traditional two-portal technique, and ABR with the single anterior portal was associated with less postoperative pain (quantity of analgesics, $200 \mathrm{mg}$ vs. $300 \mathrm{mg}$, respectively; $\mathrm{p}<0.001$ ), shorter surgical learning curve, and lower costs $(5.7 \%$ less than the two-portal technique). They also reported a higher median operative time for the standard two-portal technique $(53.5 \mathrm{~min}$ vs. $35 \mathrm{~min}$, respectively; $\mathrm{p}<0.001$ ). However, the authors found no difference in terms of clinical results between these two techniques and emphasized that, compared to the anterior two-portal technique, the single anterior portal technique prevented the instability of the glenohumeral joint due to less damage in the rotator interval. Consistent with the results obtained by Cicek et al., ${ }^{[20]}$ Ghai et al. ${ }^{[5]}$ reported similar functional outcomes, Rowe and OSIS scores, and Tegner activity level in a comparison between the anterior single-portal and anterior two-portal arthroscopic techniques. However, the operative time was significantly lower in the single anterior portal group (46.35 vs. $68.52 \mathrm{~min}$, respectively; $\mathrm{p}<0.001)$, indicating that this technique was an effective treatment modality, with similar outcomes to those of the double technique. Armangil et al. ${ }^{[21]}$ reported that $A B R$ performed using the single anterior portal technique provided comparable clinical outcomes in terms of postoperative shoulder movements with the two-portal repair technique and emphasized the importance of appropriate patient selection rather than the number of portals. Furthermore, they suggested that, compared to the 
two-portal technique, the single anterior portal technique reduced the operative time and was less invasive. A recent prospective study evaluating the effectiveness and safety of ABR revealed that, compared to two anterior portals, the single-portal technique had a shorter operative time and lower costs and that there were no significant differences between the groups in terms of clinical or functional scores, quality of life, or patient satisfaction. ${ }^{[11]}$ In the current study, we found a significant postoperative improvement in clinical and functional outcome scores compared to baseline, regardless of the number of anterior portals; however, in line with the previous findings, the single anterior portal technique had lower operative times.

Postoperative recurrent instability is the most common and undesired complication associated with ABR. ${ }^{[1]}$ Glenoid and humeral bone loss, younger age, participation in contact sports, male sex, a higher number of preoperative dislocations, and bilateral instability have been reported as the leading causes of recurrent instability. ${ }^{[21,23-26]}$ However, similar outcomes were observed in the study of Cicek et al. ${ }^{[20]}$ that compared the recurrence rate with the number of anterior portals used. In this current study, one (3.1\%) patient in Group 1 and three (7.7\%) patients in Group 2 had recurrent instability, and there were no significant differences between the groups. The overall dislocation rate in the present study was $5.6 \%$, which is consistent with that reported in the literature. ${ }^{[5,20]}$

In their study, Cicek et al. ${ }^{[20]}$ reported that the mean length of hospital stay for patients in the traditional two-portal group was significantly longer than that for patients in the single-portal group (1.5 days vs. 1 day, respectively; $\mathrm{p}<0.001$ ). However, in our patient cohort, no significant difference was observed between the two groups in terms of the length of hospital stay.

Various complications may occur during ABR, particularly during portal opening, anchor placement, and labral repair. The creation of two anterior working portals can be challenging in some populations, particularly in those with small shoulders. ${ }^{[7]}$ Iatrogenic nerve injuries and cannula breakage have been reported to occur in the standard anterior two-portal technique due to the overcrowding of cannulas in small shoulders and the difficulty associated with working in these shoulders. ${ }^{[5,7]}$ In our study, no significant complications occurred in the peri- or intraoperative period in any groups.
Studies evaluating the clinical and functional results of the single anterior portal and standard twoportal techniques for ABR are limited. Therefore, the findings of our study are valuable, supporting the existing literature by demonstrating and proving that the single anterior portal technique is safe and can reduce the operative time.

Nonetheless, this study has some limitations. First, it has a retrospective design; however, we prospectively collected the data of patients who were not lost to follow-up to obtain more accurate results. In addition, there may be a selection bias regarding which patients underwent ABR with single and two portals. Second, this study had a short follow-up period, implying that mid- or long-term functional outcomes and the recurrence rate might differ. Third, the number of patients is relatively low. Finally, we did not analyze the cost; however, in the anterior two-portal techniques, additional cannulas are used, which increases the cost of this technique compared to the single-portal technique. Further, prospective randomized-controlled studies with longer follow-up periods are warranted to obtain more accurate results regarding the effect of the number of anterior portals used in ABR on outcomes.

In conclusion, good-to-excellent clinical and functional outcomes can be obtained after ABR, regardless of the use of a single or two anterior working portals. The single anterior portal technique is as effective, reliable, and reproducible as the standard two-portal technique. In addition, the single-portal technique can reduce the operative time and the possible rate of neurovascular injury that may occur during the opening of the second anterior portal.

\section{Declaration of conflicting interests}

The authors declared no conflicts of interest with respect to the authorship and/or publication of this article.

\section{Funding}

The authors received no financial support for the research and/or authorship of this article.

\section{REFERENCES}

1. Sarıkaya B, Bozkurt C, Gül O, Bekin Sarıkaya PZ, Sipahioğlu $S$, Altay MA. Comparison of the clinical results of isolated Bankart and SLAP 5 lesions after arthroscopic repair. Jt Dis Relat Surg 2020;31:223-9.

2. Ng DZ, Lau BPH, Tan BHM, Kumar VP. Single working portal technique for knotless arthroscopic Bankart repair. Arthrosc Tech 2017;6:e1989-e1992.

3. Flinkkilä T, Knape R, Sirniö K, Ohtonen P, Leppilahti J. Long-term results of arthroscopic Bankart repair: Minimum 10 years of follow-up. Knee Surg Sports Traumatol Arthrosc 2018;26:94-9. 
4. An VV, Sivakumar BS, Phan K, Trantalis J. A systematic review and meta-analysis of clinical and patient-reported outcomes following two procedures for recurrent traumatic anterior instability of the shoulder: Latarjet procedure vs. Bankart repair. J Shoulder Elbow Surg 2016;25:853-63.

5. Ghai A, Sachdeva J, Sood M, Sud A, Chauhan M, Singh S. Similar functional outcome using single anterior portal and standard two portals technique in recurrent dislocation of shoulder. Chin J Traumatol 2020;23:102-6.

6. Castagna A, Garofalo R, Conti M, Flanagin B. Arthroscopic Bankart repair: Have we finally reached a gold standard? Knee Surg Sports Traumatol Arthrosc 2016;24:398-405.

7. Meyer M, Graveleau N, Hardy P, Landreau P. Anatomic risks of shoulder arthroscopy portals: anatomic cadaveric study of 12 portals. Arthroscopy 2007;23:529-36.

8. Maffet MW, Gartsman GM, Moseley B. Superior labrumbiceps tendon complex lesions of the shoulder. Am J Sports Med 1995;23:93-8.

9. Samilson RL, Prieto V. Dislocation arthropathy of the shoulder. J Bone Joint Surg [Am] 1983;65:456-60.

10. Shin SJ, Kim RG, Jeon YS, Kwon TH. Critical value of anterior glenoid bone loss that leads to recurrent glenohumeral instability after arthroscopic Bankart repair. Am J Sports Med 2017;45:1975-81.

11. Sebastiá-Forcada E, Martínez-Rico S, Vizcaya-Moreno MF, Lizaur-Utrilla A. Prospective study on effectiveness and safety of arthroscopic Bankart using a single anterior portal for patients with anterior shoulder instability. Rev Esp Cir Ortop Traumatol 2019;63:431-8.

12. Sugaya H, Moriishi J, Kanisawa I, Tsuchiya A. Arthroscopic osseous Bankart repair for chronic recurrent traumatic anterior glenohumeral instability. J Bone Joint Surg [Am] 2005;87:1752-60.

13. King GJ, Richards RR, Zuckerman JD, Blasier R, Dillman C, Friedman RJ, et al. A standardized method for assessment of elbow function. Research Committee, American Shoulder and Elbow Surgeons. J Shoulder Elbow Surg 1999;8:351-4.

14. Placzek JD, Lukens SC, Badalanmenti S, Roubal PJ, Freeman DC, Walleman KM, et al. Shoulder outcome measures: A comparison of 6 functional tests. Am J Sports Med 2004;32:1270-7.
15. Constant CR, Murley AH. A clinical method of functional assessment of the shoulder. Clin Orthop Relat Res 1987;(214):160-4.

16. Dawson J, Fitzpatrick R, Carr A. The assessment of shoulder instability. The development and validation of a questionnaire. J Bone Joint Surg [Br] 1999;81:420-6.

17. Skare $\varnothing$, Schrøder $\mathrm{CP}$, Mowinckel $\mathrm{P}$, Reikerås $\mathrm{O}$, Brox JI. Reliability, agreement and validity of the 1988 version of the Rowe Score. J Shoulder Elbow Surg 2011;20:1041-9.

18. Seo JB, Heo K, Yang JH, Yoo JS. Two portal technique with antegrade suture passer and knotless anchors for arthroscopic Bankart repair: A technical note. Arthroscopy and Joint Surgery 2019;6:31-4.

19. Lavender C, Lycans D, Kopiec A, Sayan A. Nanoscopic single-incision anterior labrum repair. Arthrosc Tech 2020;9:e297-e301.

20. Çiçek H, Tuhanioğlu Ü, Oğur HU, Seyfettinoğlu F, Çiloğlu O, Beyzadeoğlu T. Single anterior portal: A better option for arthroscopic treatment of traumatic anterior shoulder instability? Acta Orthop Traumatol Turc 2017;51:298-302.

21. Armangil M, Basat HÇ, Akan B, Karaduman M, Demirtaş M. Arthroscopic stabilization of anterior shoulder instability using a single anterior portal. Acta Orthop Traumatol Turc 2015;49:6-12.

22. Elena N, Woodall BM, Ahn S, McGahan PJ, Pathare NP, Shin EC, et al. Anterior shoulder stabilization using a single portal technique with suture lasso. Arthrosc Tech 2018;7:e505-e509.

23. Murphy AI, Hurley ET, Hurley DJ, Pauzenberger L, Mullett $\mathrm{H}$. Long-term outcomes of the arthroscopic Bankart repair: A systematic review of studies at 10-year follow-up. J Shoulder Elbow Surg 2019;28:2084-9.

24. Mahure SA, Mollon B, Capogna BM, Zuckerman JD, Kwon YW, Rokito AS. Risk factors for recurrent instability or revision surgery following arthroscopic Bankart repair. Bone Joint J 2018;100-B:324-30.

25. Randelli P, Ragone V, Carminati S, Cabitza P. Risk factors for recurrence after Bankart repair a systematic review. Knee Surg Sports Traumatol Arthrosc 2012;20:2129-38.

26. Horst K, Von Harten R, Weber C, Andruszkow H, Pfeifer R, Dienstknecht T, Pape HC. Assessment of coincidence and defect sizes in Bankart and Hill-Sachs lesions after anterior shoulder dislocation: A radiological study. Br J Radiol 2014;87:20130673. 